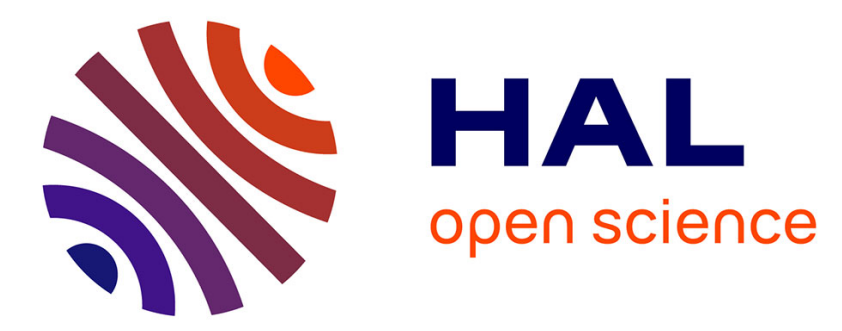

\title{
Plasticity-damage based micromechanical modelling in high cycle fatigue
}

Vincent Monchiet, Eric Charkaluk, Djimedo Kondo

\section{To cite this version:}

Vincent Monchiet, Eric Charkaluk, Djimedo Kondo. Plasticity-damage based micromechanical modelling in high cycle fatigue. Comptes Rendus Mécanique, 2006, 334 (2), pp.129-136. 10.1016/j.crme.2005.12.002 . hal-00021651

\section{HAL Id: hal-00021651 \\ https://hal.science/hal-00021651}

Submitted on 27 Jul 2021

HAL is a multi-disciplinary open access archive for the deposit and dissemination of scientific research documents, whether they are published or not. The documents may come from teaching and research institutions in France or abroad, or from public or private research centers.
L'archive ouverte pluridisciplinaire HAL, est destinée au dépôt et à la diffusion de documents scientifiques de niveau recherche, publiés ou non, émanant des établissements d'enseignement et de recherche français ou étrangers, des laboratoires publics ou privés.

\section{(c)(1)}

Distributed under a Creative Commons Attribution| 4.0 International License 


\title{
Plasticity-damage based micromechanical modelling in high cycle fatigue
}

\author{
Vincent Monchiet*, Eric Charkaluk, Djimedo Kondo \\ Laboratoire de mécanique de Lille-UMR CNRS 8107, université de sciences et technologies Lille, cité scientifique, boulevard Paul-Langevin, \\ 59655 Villeneuve d'Ascq cedex, France
}

\begin{abstract}
A micro-macro approach of multiaxial fatigue in unlimited endurance is proposed. It allows one to take into account plasticity and damage mechanisms which occur at the scale of Persistent Slip Bands (PSB). The proposed macroscopic fatigue criterion, which corresponds to microcracks nucleation at the PSB-matrix interface, is derived for different homogenization schemes (Sachs, Lin-Taylor and Kröner). The role of a mean stress and of the hydrostatic pressure in high cycle fatigue is shown; in particular, in the case of Lin-Taylor scheme and linear isotropic hardening rule at microscale, one recovers the linear dependance in pressure postulated by K. Dang Van for the macroscopic fatigue criterion. This dependence is related here to the damage micromechanism. Finally, the particular case of affine loading is presented as an illustration.
\end{abstract}

\section{Résumé}

Une modélisation micromécanique couplant plasticité et endommagement en fatigue polycyclique. On propose une approche micro-macro de fatigue multiaxiale en endurance illimitée. Elle permet de prendre en compte des mécanismes de plasticité et d'endommagement apparaissant à l'échelle des Bandes de Glissement Persistantes (BGP). Le critère macroscopique proposé, qui correspond à la nucléation de microfissures à l'interface BGP-matrice, est explicité pour différents schémas d'homogénéisation (Sachs, Lin-Taylor, Kröner). On montre qu'il rend compte du rôle de la contrainte moyenne et d'une pression hydrostatique en fatigue polycyclique; en particulier, on retrouve, dans le cas du schéma de Lin-Taylor et d'une hypothèse d'écrouissage isotrope linéaire à l'échelle microscopique, la dépendance linéaire en pression postulée par K. Dang Van pour le critère macroscopique de fatigue. Cette dépendance est liée ici au micromécanisme d'endommagement. Enfin, on présente, à titre d'illustration, le cas particulier des chargements affines.

Keywords: Fatigue; Micromechanics; Unlimited endurance; PSB

Mots-clés : Fatigue; Micromécanique; Endurance illimitée; BGP

\footnotetext{
* Corresponding author.

E-mail addresses: vincent.monchiet@ed.univ-lille1.fr (V. Monchiet), eric.charkaluk@univ-lille1.fr (E. Charkaluk), kondo@univ-lille1.fr
} (D. Kondo). 


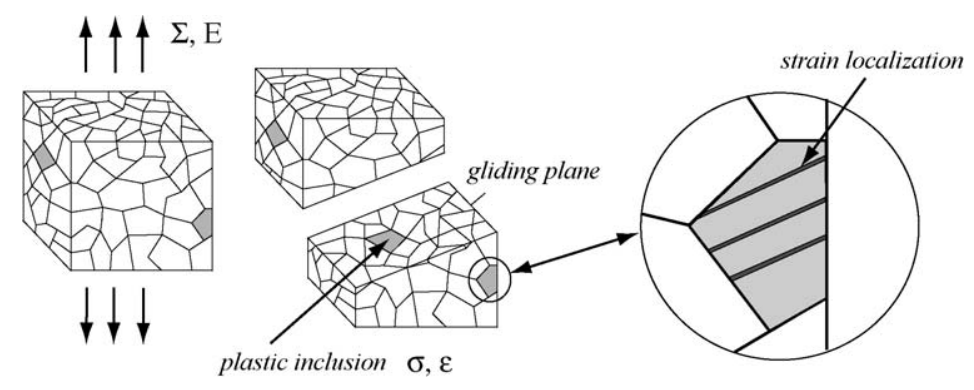

Fig. 1. Representative Volume Element (RVE).

\section{Introduction}

Structures and mechanical components submitted to cyclic loadings can fail after a finite number of cycles, even if the macroscopic response remains elastic. Fracture is the result of fatigue macrocracks nucleation and growth. Classically, in uniaxial experiments, a fatigue limit can be distinguished, i.e., there is a maximum value of the stress amplitude under which no fatigue crack can be observed whatever the applied number of cycles. The effect of a mean stress superimposed to a periodic loading has been brought to the fore by Gerber and Goodman [1] who postulated constant life diagrams as a relationship between the amplitude and the mean part of the stress.

In the case of a multiaxial stress state, several fatigue criteria have been proposed in the last few decades, using mainly macroscopic stress invariant approaches [2,3]. It has been earlier recognized that, under fatigue loadings, metals fracture is the result of the cyclic plastic strain [4] and in high cycle fatigue (HCF), at the microscale, some grains (in very low proportion), favorably oriented, undergo plasticity. This local plasticity is however confined and has no significant effect on the macroscopic response which remains elastic. Following Orowan [4], Dang Van [5] and then Papadopoulos [6] (see also [7]) formalized this multiscale framework. The resulting criterion is based on the consideration that a sufficient condition for non nucleation of fatigue cracks is to ensure elastic shakedown at all the scales. Nevertheless, plastic activity alone is not able to account for the role of pressure on the fatigue criterion. In order to take into account the mean stress effect, Papadopoulos [6] postulated a 'generalized multiaxial fatigue criterion' (which corresponds to the elastic shakedown limit) depending on the hydrostatic pressure.

In fact, the phenomenon of grain level plasticity is accompanied with the development of localized damage. The explicit incorporation of damage in HCF modelling has been the subject of several recent studies among which [8] and [9]. Although these works allow to account for the role of the pressure in fatigue, the principal criticism is that the concept of a scalar variable $d$ used to describe damage cannot be explicitly related to a particular physical micromechanism. Moreover, in the two scale model proposed by [8], no plasticity occurs at the grain scale below the macroscopic fatigue limit, and this does not correspond to experimental observations [10].

Based on experimental results obtained in the context of faced-centered-cubic structures (FCC) [11,12], and following the multiscale approach proposed by Dang Van [5] (see also [7]) in HCF, we develop a micromechanical approach of HCF criterion in which plasticity and damage are coupled at the grain scale (Section 2). Considering such coupled micro-mechanisms, the present note proposes a fatigue criterion in which an explicit dependence on the pressure and on the mean stress is shown (Section 3). Finally, through an application to affine cyclic loadings, we investigate the role of the homogenization scheme and the local hardening rule in the qualitative predictions of the approach (Section 4).

\section{Cyclic plasticity and damage at grain level}

Under low level of macroscopic loading as in the context of HCF, in the case of FCC structures, the plastic behavior is generally characterized by the activation of a predominant slip system and more precisely by the formation of strain localization band which are the potential sites for microcracks nucleation. Such bands, called persistent slip bands (PSB), have been amply studied by [11] and [12]; in particular, it is shown that dislocations concentration, which is at the origin of the damage process, is particularly important in those bands. 


\subsection{Monocrystal plasticity}

The predominant activated slip system is characterized by a plane, defined by a unit normal vector $\underline{n}$ and a slip direction $\underline{m}$. Let us introduce $\sigma$ and $\boldsymbol{\varepsilon}$ respectively the microscopic (i.e., at the grain level) stress and strain fields. As classically, an additive decomposition of the total strain $\boldsymbol{\varepsilon}$ into elastic strain, $\boldsymbol{\varepsilon}^{e}$, and plastic strain, $\boldsymbol{\varepsilon}^{p}$, at the microscopic scale is adopted: $\boldsymbol{\varepsilon}=\boldsymbol{\varepsilon}^{e}+\boldsymbol{\varepsilon}^{p}$. The monocrystal plastic behavior is described by the plastic criterion $f$ corresponding to Schmid's law, written in the classical form:

$$
f=|\tau-x|-\tau_{0}-R
$$

$\tau$ represents the shear stress acting on the slip plane: $\tau=\sigma: \Delta, \Delta$ being the second order symmetric tensor $\Delta=$ $\underline{n} \stackrel{s}{\otimes} \underline{m}=\frac{1}{2}(\underline{n} \otimes \underline{m}+\underline{m} \otimes \underline{n}) ; \tau_{0}$ is the critical shear stress. $x$ and $R$ are the hardening variables defining respectively:

- A kinematic linear rule: $x=c \gamma^{p}$, where $\gamma^{p}$ is such that: $\varepsilon^{p}=\gamma^{p} \boldsymbol{\Delta}$.

- An isotropic linear or non linear rule:

$$
\left\{\begin{array}{l}
R(p)=R_{0} p \\
R(p)=R_{s}\{1-\exp (-r p)\}
\end{array}\right.
$$

$p$ is the cumulated plastic slip such that: $p=\int_{0}^{t}\left|\dot{\gamma}^{p}\left(t^{\prime}\right)\right| \mathrm{d} t^{\prime} ; R_{0}, R_{s}$ and $r$ are three parameters.

\subsection{Microdamage within localization bands}

The strain localization into the PSBs is also accompanied by a dislocations annihilation mechanism. Transmission electron microscopy studies [12] reveal that there is a critical spacing between dislocations at which their annihilation is favored. Dislocations will annihilate to form a vacancy or an interstitial point defect. According to Essmann et al. [12], vacancies production is the dominant points defects generation process. The transition from vacancy production to the formation of microvoids or microcracks along the PSBs is not yet well understood. Therefore, we assume that the formation of these microvoids is the result of the agglomeration and the growth of vacancies formed by the dislocation annihilation process. It follows that damage along PSBs is the result of both two mechanisms: vacancies production and voids growth which is the result of the combined effect of the slip-like plastic activity and pressure.

A phenomenological model for points defects production by dislocations annihilation has been already proposed by Essmann et al. [11]. For this purpose, introducing two parameters $\rho_{0}$ and $k_{a}$, let us first define the dislocation density, $\rho_{p}$, and the annihilation density, $\rho_{a}$ :

$$
\rho_{p}=\rho_{0} k_{a} p ; \quad \rho_{a}=\rho_{0}\left\{k_{a} p-1+\exp \left(-k_{a} p\right)\right\}
$$

Noting that dislocations annihilation is not an irreversible mechanism (points defects could be mutually annihilated), a points defects density law $\dot{\rho}_{d}$ was introduced:

$$
\dot{\rho}_{d}=\dot{\rho}_{a}-k_{e} \rho_{d} \dot{p}
$$

Following [12], it is assumed that all points defects are of vacancy-type. Denoting by $V_{0}$ the volume occupied by a vacancy, integrating (4) and taking into account (3), the porosity associated to the mechanism of vacancies production, $\eta_{a}$, is given by:

$$
\eta_{a}(p)=V_{0} \rho_{d}=\frac{A_{0} k_{a}}{k_{e}\left(k_{a}-k_{e}\right)}\left\{k_{a}\left\{1-\exp \left(-k_{e} p\right)\right\}-k_{e}\left\{1-\exp \left(-k_{a} p\right)\right\}\right\}
$$

with $k_{e}$ a constant and $A_{0}=V_{0} \rho_{0}$. For very large value of $p, \eta_{a}$ reaches a limit $\eta_{a}^{s}=A_{0} k_{a} / k_{e}$ : there is an equilibrium between points defects annihilation and production. Let us introduce the total porosity at the grain scale:

$$
\eta=\eta_{a}+\eta_{g}
$$

where $\eta_{g}$ is the porosity associated to the mechanism of voids growth as previously mentioned.

As voids growth induces volume change, the plastic strain at the grain scale can be decomposed in the following form, 1 being the first order identity tensor:

$$
\boldsymbol{\varepsilon}^{p}=\gamma^{p} \boldsymbol{\Delta}+\varepsilon_{h}^{p} \mathbf{1}
$$


where the volumetric plastic strain $\varepsilon_{h}^{p}$, due to voids growth and deduced from the mass balance equation, is given by:

$$
\eta_{g}=1-\exp \left(-3 \varepsilon_{h}^{p}\right)
$$

As a first approximation, voids growth is described by means of a modified Rice and Tracey-type model [13]. For this purpose, we assume spherical voids and for simplicity we replace the crystal by an equivalent von Mises material. The proposed modification leads to (see Appendix A.7):

$$
\dot{\varepsilon}_{h}^{p}=\frac{1}{2 \sqrt{3}} \eta \sinh \left\{\frac{\sqrt{3}}{2} \frac{\sigma_{h}}{\tau_{0}}\right\} \dot{p}
$$

where $\sigma_{h}=\operatorname{tr} \sigma / 3$.

A local approach of fatigue crack nucleation at the PSB-matrix interface consists then in introducing a critical value of the porosity, $\eta_{c}$ :

$$
\eta_{a}+\eta_{g}=\eta_{c}
$$

The damage-dependent criterion (10) is the threshold below which no crack nucleation could be observed. From (5), (8) and (9), it is readily seen that this criterion depends explicitly on the hydrostatic pressure $\sigma_{h}$, on the cumulated plastic strain $p$ and consequently on the local shear stress $\tau$.

\section{Micro-macro based determination of the fatigue criterion}

\subsection{Expression of the local fatigue criterion}

For the determination of the fatigue criterion, following [5], we assume that a necessary condition for non nucleation of cracks is that the monocrystal shakedowns elastically. For [6] (see [7]), this is also a sufficient condition; then, elastic shakedown limit coincides with the fatigue limit. In order to take into account the dependence on the hydrostatic pressure, a 'generalized multiaxial fatigue limit' has been postulated by [6].

In our HCF approach, elastic shakedown is a necessary but not a sufficient condition for non nucleation of cracks: in the elastic shakedown regime, a crack may nucleate if a critical porosity along the PSB-matrix interface is attained (see condition (10)). Therefore, as the grain shakedowns elastically, $p$ is bounded and so the porosity is very low; the following approximations can then be made:

(i) $\eta_{g} \ll 1$, then mass balance equation (8) reads: $\eta_{g} \simeq 3 \varepsilon_{h}^{p}$.

(ii) $\eta_{a}$ is low compared to its saturation value $\eta_{a}^{s}$; then, point defect annihilation process is neglected $\left(k_{e}=0\right)$ particularly in Eq. (4).

Taking into account these approximations in (5) and (8), the local fatigue criterion, deduced from (10), reads:

$$
A_{0}\left(k_{a} p-1+\exp \left(-k_{a} p\right)\right)+3 \varepsilon_{h}^{p}<\eta_{c}
$$

Since $p$ and $\varepsilon_{h}^{p}$ are defined at the grain scale, in order to establish a macroscopic expression of the fatigue criterion, a non linear homogenization approach is due.

\subsection{The macroscopic fatigue criterion}

Let us first recall that the macroscopic behavior remains elastic. Therefore, the macroscopic stress tensor, $\boldsymbol{\Sigma}$, and the macroscopic elastic strain tensor, $\boldsymbol{E}$, are given by: $\boldsymbol{\Sigma}=\mathbb{C}: \boldsymbol{E}$ where $\mathbb{C}$ is the stiffness tensor of the matrix. For simplicity, $\mathbb{C}$ is assumed isotropic: $\mathbb{C}=3 k \mathbb{J}+2 \mu \mathbb{K}, k$ and $\mu$ being respectively the bulk and shear modulus of the matrix, $\mathbb{J}=\frac{1}{3} \mathbf{1} \otimes \mathbf{1}$ and $\mathbb{K}=\mathbb{I}-\mathbb{J}$ with $\mathbb{I}$ the fourth order symmetric identity tensor.

Moreover, as a first approximation, it is assumed that the elastic behavior of the continuum and of its constituent grains are the same and reads $\sigma=\mathbb{C}: \varepsilon^{e}$.

As a first approach, Lin-Taylor or Sachs models could be considered:

$$
\begin{array}{ll}
\boldsymbol{\sigma} & =\boldsymbol{\Sigma}-\mathbb{C}: \boldsymbol{\varepsilon}^{p} \quad \text { (Lin-Taylor) } \\
\boldsymbol{\sigma} & =\boldsymbol{\Sigma} \quad(\text { Sachs })
\end{array}
$$


A more elaborated, but simple, scheme which can be used to describe crystalline plasticity is the self consistent model (SCM) of Kröner [14] (see also [15]). In the particular case of high cycle fatigue, the plastic strain being confined into a few number of grains embedded in the elastic matrix, the overall behavior is close to the one of polycrystals near the yield limit. For such situation, the Kröner SCM is suitable and gives:

$$
\boldsymbol{\sigma}=\boldsymbol{\Sigma}-\mathbb{C}:(\mathbb{I}-\mathbb{P}: \mathbb{C}): \boldsymbol{\varepsilon}^{p}
$$

where $\mathbb{P}$ is the fourth order Hill tensor; considering a spherical inclusion, $\mathbb{P}$ reads:

$$
\mathbb{P}=\frac{a}{3 k} \mathbb{J}+\frac{b}{2 \mu} \mathbb{K} \quad \text { with } a=\frac{3 k}{3 k+4 \mu} \text { and } b=\frac{6}{5} \frac{k+2 \mu}{3 k+4 \mu}
$$

The interaction laws, given by (12) and by (13) with (14) can be put in the general following form:

$$
\sigma=\Sigma-\mathbb{C}^{*}: \varepsilon^{p}
$$

with $\mathbb{C}^{*}=0$ for Sachs's model, $\mathbb{C}^{*}=\mathbb{C}$ for Lin-Taylor's model and $\mathbb{C}^{*}=\mathbb{C}:(\mathbb{I}-\mathbb{P}: \mathbb{C})$ for Kröner scheme. ${ }^{1}$

It follows that the hydrostatic pressure $\sigma_{h}$ and the shear stress $\tau$, which enter into the local criterion, (10) are given by:

$$
\tau=\boldsymbol{\Sigma}: \Delta-2 \mu^{*} \gamma^{p} ; \quad \sigma_{h}=\Sigma_{h}-3 k^{*} \varepsilon_{h}^{p}
$$

with $\mu^{*}=\mu(1-b), k^{*}=k(1-a)$.

Lin-Taylor estimate is obviously recovered for $a=b=0$ and the Sachs model for $a=b=1$.

As previously shown, the translation at the macroscopic level of the fatigue criterion (11) requires the expression of $p$ and $\varepsilon_{h}^{p}$ as functions of the macroscopic fields. $p$ is related to $\tau$ by means of the Schmid's law (1) and then to the macroscopic stress by the interaction law (16). $\varepsilon_{h}^{p}$ is obtained by integrating (9) in which the expression of $\sigma_{h}$, given by (16), is used:

$$
\dot{\varepsilon}_{h}^{p}=\frac{1}{2 \sqrt{3}} \eta \sinh \left\{\frac{\sqrt{3}}{2} \frac{\Sigma_{h}-3 k^{*} \varepsilon_{h}^{p}}{\tau_{0}}\right\} \dot{p}
$$

In a general loading case, $p$ and $\varepsilon_{h}^{p}$ can be determined by using a numerical procedure. For an illustration purpose, we consider now the particular case of affine loadings.

\section{Applications to affine loadings}

The macroscopic stress tensor, $\boldsymbol{\Sigma}(t)$, associated to an affine loading, is decomposed into an alternative and a mean part: $\boldsymbol{\Sigma}(t)=\boldsymbol{\Sigma}_{a} \sin (\omega t)+\boldsymbol{\Sigma}_{m}$.

When the grain shakes down elastically, the mean shear stress and the amplitude of the shear stress are given, using (1), by: $\tau_{m}=c \gamma_{m}^{p}$ and $\tau_{a}=\tau_{0}+R(p)$. The interaction law (16), for the affine loading, reads:

$$
\boldsymbol{\Sigma}_{m}: \Delta=\left(c+\mu^{*}\right) \gamma_{m}^{p} ; \quad \boldsymbol{\Sigma}_{a}: \Delta=\tau_{0}+R(p)
$$

Consider a first situation of an alternated torsion with a mean torsion: $T(t)=T_{a} \sin (\omega t)+T_{m}$. Using (18), $p$ and $\gamma_{m}^{p}$, are given, in the favorably oriented grain, by:

$$
\gamma_{m}^{p}=\frac{T_{m}}{c+\mu^{*}} ;\left\{\begin{array}{l}
p=\frac{T_{a}-\tau_{0}}{R_{0}} \quad \text { (For the linear isotropic hardening rule) } \\
p=\frac{1}{r} \ln \left\{\frac{R_{s}}{R_{s}+\tau_{0}-T_{a}}\right\} \quad \text { (For the non-linear isotropic hardening rule) }
\end{array}\right.
$$

Since $\Sigma_{h}=0$, one has $\varepsilon_{h}^{p}=0$ and $\eta_{g}=0$. Consequently, the fatigue criterion (11) is found to depend only on the variable $p$; then a mean torsion has no effect on the fatigue limit which is consistent with experimental data provided by [3].

1 An extension to the anisotropic behavior can be made, following a recent work of [16]. 

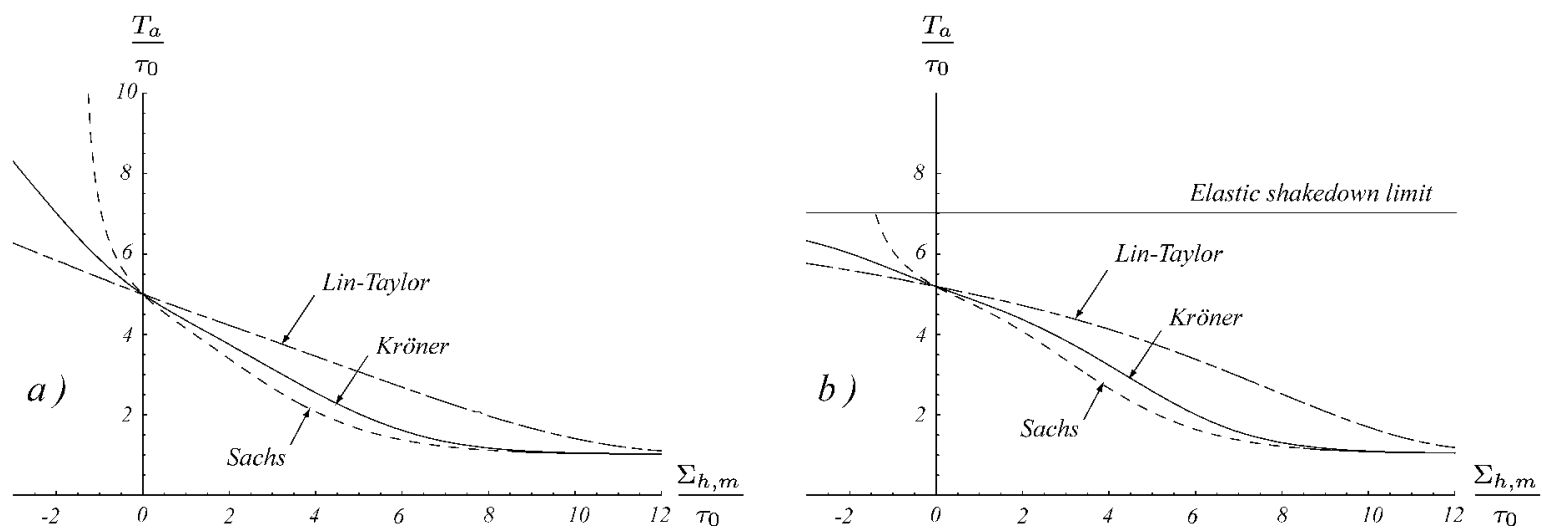

Fig. 2. Application of the fatigue criterion in particular case of an alternated torsion loading with a mean pressure, $T_{a} / \tau_{0}$ versus $\Sigma_{h, m} / \tau_{0}:($ a) linear isotropic hardening; (b) non-linear isotropic hardening. Parameters used are $k=70000 \mathrm{MPa}, v=0.33, A_{0}=2 \times 10^{-4}, k_{a}=20, R_{0}=350 \mathrm{MPa}$, $R_{S}=420 \mathrm{MPa}, r=1.5, \tau_{0}=70 \mathrm{Mpa}, \eta_{c}=0.003$.

Let us consider now a second case corresponding to an alternated torsion loading with a mean pressure $\left(\boldsymbol{\Sigma}_{m}=\right.$ $\left.\Sigma_{h, m} \mathbf{1}\right)$. For Sachs model, a closed form expression of the fatigue criterion is found:

$$
\frac{2}{\sqrt{3}} \frac{A_{0} k_{a}}{H_{m}\left(\sqrt{3} H_{m}+2 k_{a}\right)}\left\{2 k_{a}\left\{\exp \left\{\frac{\sqrt{3}}{2} H_{m} p\right\}-1\right\}+\sqrt{3} H_{m}\left\{\exp \left\{-k_{a} p\right\}-1\right\}\right\}<\eta_{c}
$$

with $H_{m}=\sinh \left\{\frac{\sqrt{3}}{2} \frac{\Sigma_{h, m}}{\tau_{0}}\right\}$. For Lin-Taylor model, numerical resolution of Eq. (17) shows that, for moderate values of the pressure, $\varepsilon_{h}^{p}$ reaches a saturation state corresponding to $\dot{\varepsilon}_{h}^{p}=0$, therefore, $\sigma_{h, m}=\Sigma_{h, m}-3 k \varepsilon_{h}^{p}=0$ and $\exp \left(-k_{a} p\right)$ can be neglected in (11). An analytical expression of the criterion is then obtained:

$$
A_{0} k_{a} p+\frac{\Sigma_{h, m}}{k}<A_{0}+\eta_{c}
$$

It is worth noticing that, for a linear isotropic hardening assumption (see Eq. (19)), the fatigue criterion for the LinTaylor scheme, reads:

$$
\frac{T_{a}}{\tau_{0}}+\alpha \frac{\Sigma_{h, m}}{\tau_{0}}<\beta ; \quad \text { with } \alpha=\frac{R_{0}}{A_{0} k_{a} k} ; \beta=\frac{R_{0}}{A_{0} k_{a} \tau_{0}}\left\{\eta_{c}+A_{0}\left\{1+\frac{\tau_{0} k_{a}}{R_{0}}\right\}\right\}
$$

This expression is in agreement with the fatigue criterion proposed by [5]. $\beta \tau_{0}$ corresponds to the fatigue limit in alternated torsion.

Fig. 2 shows representation of the fatigue criterion for an alternated torsion with a mean pressure and for the different homogenization schemes. Figs. 2(a) and (b) correspond respectively to a linear and a non linear isotropic hardening. For the two hardening rules, the three homogenization schemes lead to different predictions, the Kröner model being between the two others. Besides the influence of the homogenization scheme and of the hardening rule, the results show a remarkable effect of the pressure on the fatigue criterion is shown. However, for negative pressure, the results of Sachs approach seem to be physically inconsistent. All those qualitative results have now to be confirmed by a comparison with experimental existing data.

\section{Conclusion}

A multiscale approach for the determination of a High Cycle Fatigue criterion has been proposed. It allows us to take into account, not only plasticity activity in some grains, but also damage due to microvoid growth along the PSB-matrix interface. The proposed criterion shows a significant effect of the mean pressure which is due to the considered damage mechanism. It predicts also that a mean shear stress has no effect on the fatigue limit; this qualitative prediction is in agreement with experimental data. 


\section{Appendix A. A modified Rice and Tracey void growth model}

The objective of this appendix is to derive a new approximation of a void growth model for arbitrary value of triaxiality. For this purpose, let us consider a spherical cavity (radius $R$ ), in an infinite perfectly plastic medium which is subjected to a uniform remote strain rate field $\dot{\boldsymbol{\varepsilon}}^{\infty}$. Let us denote by $r, \theta, \phi$, the spherical coordinates and $\underline{e}_{r}$ the radial unit vector. The strain rate field is decomposed in two terms, the remote field and a strain rate field (related to multiple slip), $\dot{\boldsymbol{\varepsilon}}^{D}$, which allows us to take into account void expansion: $\dot{\boldsymbol{\varepsilon}}=\dot{\boldsymbol{\varepsilon}}^{\infty}+\dot{\boldsymbol{\varepsilon}}^{D}$, with $\dot{\boldsymbol{\varepsilon}}^{\infty}=\dot{\gamma} \boldsymbol{\Delta}$. In order to apply the Rice and Tracey [13] result, we propose to replace the monocrystal by an equivalent von Mises material; this is justified by the activation of multiple slip systems around the cavity. Moreover, we consider a spherically symmetric strain void expansion field: $\dot{\boldsymbol{\varepsilon}}^{D}=\frac{R^{3}}{r^{3}} \dot{\varepsilon}_{\mathrm{eq}}^{\infty}\left(\mathbf{1}-3 \underline{e}_{r} \otimes \underline{e}_{r}\right)\left(\dot{\varepsilon}_{\mathrm{eq}}^{\infty}=\sqrt{\frac{2}{3} \dot{\boldsymbol{\varepsilon}}^{\infty}: \dot{\boldsymbol{\varepsilon}}^{\infty}}=\frac{1}{\sqrt{3}} \dot{p}\right)$. Therefore, the void growth law reads:

$$
\frac{\dot{R}}{R}=D \dot{\varepsilon}_{\mathrm{eq}}^{\infty}=D \frac{\dot{p}}{\sqrt{3}}
$$

where, $\sigma_{0}$ being the yield stress of the equivalent von Mises material, such that $\sigma_{0} \dot{\varepsilon}_{e q}^{\infty}=\tau_{0} \dot{p}$, i.e., $\sigma_{0}=\sqrt{3} \tau_{0}, D$ is solution of (see [13]):

$$
\int_{z=0}^{z=1} \frac{1}{4 \pi} \int_{S}\left\{\frac{2 D z-m}{\left\{1-4 m D z+4 D^{2} z^{2}\right\}^{1 / 2}}+m\right\} \mathrm{d} S \frac{\mathrm{d} z}{z}=\frac{2}{3} \frac{\sigma_{h}}{\sigma_{0}}
$$

In (A.2), $z=\frac{R^{3}}{r^{3}}$ and $m=\frac{1}{\dot{\varepsilon}_{\mathrm{eq}}^{\infty}} \dot{\boldsymbol{\varepsilon}}^{\infty}: \underline{e}_{r} \otimes \underline{e}_{r}(-1 / 2<m<1 / 2)$. The inner integration is over the unit sphere with $\mathrm{d} S=\sin (\phi) \mathrm{d} \phi \mathrm{d} \theta$. Since (A.2) is not analytically integrable, Rice and Tracey proposed an approximation for high value of $D$, which is valid for large value of $\left|\frac{\sigma_{h}}{\sigma_{0}}\right|$ :

$$
D=\frac{1}{4} \exp \left\{\frac{3}{2} \frac{\sigma_{h}}{\sigma_{0}}+c\right\} \quad \text { with } c=\frac{1}{4 \pi} \int_{S}(\varepsilon-m) \ln (1-\varepsilon m) \mathrm{d} S \text { and } \varepsilon=\operatorname{sign}\left\{\frac{\sigma_{h}}{\sigma_{0}}\right\}
$$

We propose here a new approximation for any value of $\frac{\sigma_{h}}{\sigma_{0}}$. For this purpose, we develop (A.2), around $m=0$, in a first time:

$$
\int_{z=0}^{z=1} \frac{1}{4 \pi} \int_{S}\left\{\frac{2 z D}{\sqrt{1+4 z^{2} D^{2}}}+\left\{1-\frac{1}{\left(1+4 z^{2} D^{2}\right)^{3 / 2}}\right\} m-\frac{2 z D\left(1-2 z^{2} D^{2}\right)}{\left(1+4 z^{2} D^{2}\right)^{5 / 2}} m^{2}+\mathrm{o}\left(m^{3}\right)\right\} \mathrm{d} S \frac{\mathrm{d} z}{z}=\frac{3}{2} \frac{\sigma_{h}}{\sigma_{0}}
$$

Using then the following relations: $\frac{1}{4 \pi} \int_{S} \mathrm{~d} S=1, \frac{1}{4 \pi} \int_{S} m \mathrm{~d} S=0, \frac{1}{4 \pi} \int_{S} m^{2} \mathrm{~d} S=\frac{1}{5}$, the integration of (A.2) gives:

$$
\operatorname{arcsinh}(2 D)-\frac{2 D}{5} \frac{1+2 D^{2}}{\left(1+4 D^{2}\right)^{3 / 2}}=\frac{3}{2} \frac{\sigma_{h}}{\sigma_{0}}
$$

Since the second term of the left hand of (A.5) is bounded $\left(0<\frac{2 D}{5} \frac{1+2 D^{2}}{\left(1+4 D^{2}\right)^{3 / 2}}<\frac{1}{10}\right)$ and low compared to $\operatorname{arcsinh}(2 D)$, it is neglected in the present study. Then $D$ is given by:

$$
D=\frac{1}{2} \sinh \left\{\frac{3}{2} \frac{\sigma_{h}}{\sigma_{0}}\right\}=\frac{1}{2} \sinh \left\{\frac{\sqrt{3}}{2} \frac{\sigma_{h}}{\tau_{0}}\right\}
$$

The plastic strain related to the cavity growth, is given by: $\dot{\varepsilon}_{h}^{p}=\frac{1}{3} \frac{\dot{\Omega}}{\Omega}$, in which $\Omega$ is the volume of the monocrystal. Considering cavities with an average volume $\omega\left(\omega=\frac{4 \pi}{3} R^{3}\right)$, in an incompressible matrix, the mass balance equation, $\dot{\omega}=\dot{\Omega}$, reads (using (A.1)):

$$
\dot{\varepsilon}_{h}^{p}=\frac{1}{3} \frac{\dot{\Omega}}{\Omega}=\eta \frac{\dot{R}}{R}=\frac{1}{2 \sqrt{3}} \eta \sinh \left\{\frac{\sqrt{3}}{2} \frac{\sigma_{h}}{\tau_{0}}\right\} \dot{p}
$$




\section{References}

[1] S. Suresh, Fatigue of Materials, Cambridge University Press, Cambridge, UK, 2001.

[2] B. Crossland, Effect of large hydrostatic pressures on the torsional fatigue strength of an alloy steel, in: Proc. Int. Conf. on Fatigue of MetalsI.M.E.-U.K., 1956, pp. 138-149.

[3] G. Sines, Behavior of metals under complex static and alternating stresses, in: G. Sines, J.L. Waisman (Eds.), Metal Fatigue, McGraw-Hill, New York, 1959, pp. 145-159.

[4] E. Orowan, Theory of the fatigue of metals, in: Proceedings of the Royal Academy A, London, 1939, pp. 78-106.

[5] K. Dang Van, Sur la résistance à la fatigue des métaux, Ph.D. Thesis, University Paris 6, 1971.

[6] I.V. Papadopoulos, Fatigue polycyclique des métaux : une nouvelle approche, Ph.D. Thesis, ENPC, 1987.

[7] K. Dang Van, I.V. Papadopoulos (Eds.), High Cycle Metal Fatigue, From Theory to Applications, in: K. Dang Van, I.V. Papadopoulos (Eds.), CISM Courses and Lectures, no. 392.

[8] J. Lemaitre, J.P. Sermage, R. Desmorat, A two scale damage concept applied to fatigue, Int. J. Fracture 97 (1999) 67-81.

[9] L. Flacelière, Contribution à la modélisation du dommage en fatigue multiaxiale d'un acier C36 — confrontation à l'expérience, Ph.D. Thesis, University of Poitiers, 2004

[10] P. Cugy, A. Galtier, Microplasticity and temperature increase in low carbon steels, in: A.F. Blom (Ed.), Proceedings of the 8th International Fatigue Congress, Stockholm, 2002.

[11] U. Essmann, H. Mughrabi, Annihilation of dislocations during tensile and cyclic deformation and limits of dislocation densities, Philosophical Magazine A 40 (6) (1979) 731-756.

[12] U. Essmann, U. Gosele, H. Mughrabi, A model of extrusions and intrusions in fatigued metals. I. Point-defects production and the growth of extrusions, Philosophical Magazine A 44 (2) (1991) 405-426.

[13] J.R. Rice, D.M. Tracey, On the ductile enlargement of voids in triaxial stress fields, J. Mech. Phys. Solids 17 (1969) $201-217$.

[14] E. Kröner, Zur plastichen verformung des vielkristalls, Acta Metall. 99 (1961) 155-161.

[15] M. Bornert, T. Bretheau, P. Gilormini, Homogénéisation en mécanique des matériaux, Tome 2, Comportements non linéaires et problèmes ouverts, Hermes Science, 2001.

[16] F. Cano, A. Constantinescu, H. Maitournam, Critère de fatigue polycyclique pour des matériaux anisotropes: application aux monocristaux, C. R. Mécanique 332 (2004) 115-121. 